\title{
Building Extraction of Polarimetric SAR Image Based on Associated Feature and SVM
}

\author{
Yuting Sheng ${ }^{1,2}$, Zheng Zhao ${ }^{1,2, *}$, Huan Chen ${ }^{1,2}$ \\ ${ }^{1}$ College of Geomatics, Shandong University of Science and Technology, Qingdao 266590, China - 1179543358@qq.com, \\ zhaozheng@casm.ac.cn, chcx0329@163.com \\ ${ }^{2}$ Key Laboratory of Geo-Informatics of State Bureau of Surveying and Mapping, Chinese Academy of Surveying and Mapping, \\ 100830 Beijing, China - guhy@casm.ac.cn
}

KEY WORDS: Building extraction, GF-3, Normalized Circular-pol Correlation Coefficient, Texture features, Gray Level Cooccurrence Matrix

\begin{abstract}
:
The identification and extraction of building is a vital task in urban environmental planning and research. High-resolution SAR technology has the advantages of all-day, all-weather and strong penetration, and has become one of the important technical means to study urban areas. A novel method for building extraction using both associated features and SVM that based on the full polarization SAR image of GF-3 is proposed in this paper. Firstly, filtering the GF-3 image to reduce the speckles of the image, then texture features based on Span map are extracted by using GLCM. Principal component analysis is used to remove the correlation between them and select the best texture features. The normalized circular-pol correlation coefficient is introduced as the polarization feature and combines with the best texture features. Finally, the image is classified and extracted by SVM. In this paper, the extraction results are compared with the results of the texture feature building extraction method. The experimental results show that the proposed method can obtain higher extraction precision, and the extraction effect is obviously optimized.
\end{abstract}

\section{INTRODUCTION}

With the development of the national economy, the development of urban areas in China has been increasingly promoted, and urban construction and residential land use have received more and more attention. High-resolution synthetic aperture radar technology has the characteristics of fast, wide range and high timeliness, and has become one of the important technical means to study urban areas. The high-resolution SAR technology has the advantages of all day, all weather and has certain penetrating power to the terrain. It has great advantages for the recognition and extraction of SAR image objects, making SAR technology have received much attention in urban extraction, urban monitoring and disaster assessment. At present, urban building extraction has become an important topic in the field of SAR image interpretation, laying a foundation for future national land surveys, urban planning and other applications.

People usually judge different ground objects according to the gray scale difference of the image, but it is difficult to distinguish some images with similar gray scale but not the same object, which is easy to produce "same spectrum foreign material" phenomenon. High-resolution SAR images contain rich texture information. Different ground objects have different texture features due to different surface roughness and directionality, which plays an important role in improving the accuracy of terrain classification and information extraction. Therefore, more and more domestic and foreign scholars have used texture analysis methods to carry out research on urban building extraction of SAR images. For example, some people used Radarsat images to effectively classify urban regional targets (Ban, Hu, 2007). It perform texture analysis based on gray level co-occurrence matrix and achieved good classification results (Dell, Gamba, 2003). To get better classification results, the method has effectively classified water bodies and residential areas with the Radarsat-1 SAR image based on the gray level co-occurrence matrix algorithm $(\mathrm{Hu}$, 2008). It has the advantage of used the ALOS-2 data to extract $87.2 \%$ of earthquake-damaged buildings using texture feature correlation detection method ( $\mathrm{Li}, 2018)$. Compared with traditional unipolar data, it has extracted $82 \%$ of buildings through AIRSAR's L-band full polarization data (Sun, 2013). Urban construction and residential land use have received more and more attention, this method fine-classified urban areas with high-resolution airborne images based on Markov random field texture analysis (Tison, 2004). Different from traditional methods, it used a COSMO-SkyMed SAR image with a spatial resolution of $3 \mathrm{~m}$ to effectively extracted the architectural regions in two sub-regions by using gray and texture features. The extraction effect is significantly better than the method of not using texture features (Xu, 2012). Another method used a variogram-based method to extract buildings with airborne SAR images with a resolution of $1 \mathrm{~m}$ (Zhao, 2009). Some scholars use airborne P-band PolSAR images with a resolution of $1 \mathrm{~m}$ to extract urban collapsed buildings based on texture features of gray-scale co-occurrence matrix (Zhai, 2016).

According to the above analysis, the use of high-resolution spaceborne or airborne SAR images for building extraction has become a research hotspot. However, there are few studies on the use of the GF-3 satellite launched by China to extract buildings. Moreover, most of the above studies just use texture features to extract buildings, and the features are single. Therefore, based on GF-3 image, the paper first extracts the texture features on the Span map, then the normalized circular polarization correlation coefficient is introduced as the

\footnotetext{
* Corresponding author at: Key Laboratory of Geo-Informatics of State Bureau of Surveying and Mapping, Chinese Academy of Surveying and Mapping, Beijing.

E-mail address: zhaozheng@casm.ac.cn.
} 
polarization feature and combined with the best texture features after screening. Finally, the image is classified and extracted based on SVM. The results of this method are compared with the results of the texture feature extraction methods. It shows that the proposed method can effectively extract buildings, and the extraction accuracy of buildings is significantly improved compared with the latter.

\section{FEATURE SELECTION}

\subsection{Texture feature}

The statistical method of the gray level co-occurrence matrix was proposed in the early 1970s (Haralick, 1973). They defined 14 texture feature parameters based on gray level co-occurrence matrix. The definition of the gray level co-occurrence matrix is as follows: Starting from the pixel where the image $(\mathrm{x}, \mathrm{y})$ has a gray scale of $i$, the probability $P(i, j, d, \theta)$ at which the pixel $(x+a$, $\mathrm{y}+\mathrm{b}$ ) whose distance is $\mathrm{d}$, the direction is $\theta$, and the gradation is $\mathrm{j}$ is simultaneously counted. as the picture shows:

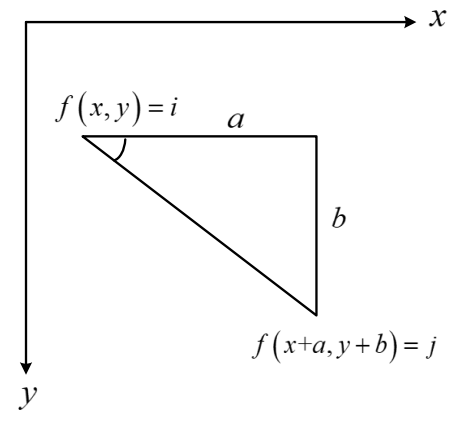

Figure 1. Gray Level Co-occurrence Matrix

Its mathematical formula is:

$$
P(\mathrm{i}, \mathrm{j}, \quad d, \theta)=\left\{\begin{array}{l}
{[(x, y),(x+a, y+b)] \mid f(x, y)=i, f(x+a, y+b)=j ;} \\
x=0,1,2 \cdots M ; y=0,1,2 \cdots N
\end{array}\right\}
$$

where $\quad \mathrm{i}, \mathrm{j}=$ row and column numbers of the gray level cooccurrence matrix

$\mathrm{x}, \mathrm{y}=$ pixel coordinates in the image

$\mathrm{M}, \mathrm{N}=$ the number of rows and columns

$\theta=$ the angle of the two-pixel connection vector

$\mathrm{d}=$ the distance between two pixels

A large number of texture features can be calculated based on the gray level co-occurrence matrix, which can assist the highresolution SAR image for feature classification and information extraction according to the selected texture features. Commonly used texture feature statistical parameters include: mean, contrast, entropy, homogeneity, etc. Definition is as follows:

(1) Mean

$$
\text { mean }=\sum_{i=0}^{L-1} \sum_{j=0}^{L-1} i g(i, j)
$$

Mean reflects the degree of regularity of the texture.

$$
\text { con }=\sum_{i=0}^{L-1} \sum_{j=0}^{L-1}(i-j)^{2}[g(i, j)]
$$

Contrast reflects the sharpness of the image and the depth of the texture. The larger the contrast value, the deeper the groove and the higher the texture definition.

(3) Entropy

$$
e n t=\sum_{i=0}^{L-1} \sum_{j=0}^{L-1} g(i, j) \log (g(i, j))
$$

Entropy is a measure of the randomness of information contained in an image, reflecting the complexity of the gray distribution of the image.

(4) Homogeneity

$$
\text { hom }=\sum_{i=0}^{L-1} \sum_{j=0}^{L-1} \frac{g(i, j)}{1+|i-j|}
$$

Homogeneity reflects the uniformity of the image.

The mathematical expression of $\mathrm{g}(i, j)$ in each formula is:

$$
g(i, j)=\frac{P(i, j, d, \theta)}{\sum_{i=0}^{L-1} \sum_{j=0}^{L-1} P(i, j, d, \theta)}
$$

where $P(i, j, d, \theta)=$ the times of $\mathrm{i}$ and $\mathrm{j}$ two pixels appear together in gray level co-occurrence matrix

$\mathrm{L}=$ the gray level of the image

$g(i, j)=$ the values of the $\mathrm{i}$-th row and the $\mathrm{j}$-th

column of the normalized gray-scale co-occurrence matrix that given the $\mathrm{d}$ and $\theta$

\subsection{Normalized Circular-Pol Correlation Coefficient}

In polarimetric SAR images, the backscattering of buildings is quite different from the backscattering of natural features. Backscattering of natural features is often reflectively symmetric, which is $S_{h v}^{*} S_{h h} \approx S_{h v}^{*} S_{v v} \approx 0$. Buildings are nonreflective symmetry targets, $S_{h v}^{*} S_{h h}=S_{h v}^{*} S_{v v} \neq 0$. Specific reference parameters and formula derivation reference (Yang, 2012), it not elaborated here. In the classification and extraction of SAR image features, some inclined buildings are often misinterpreted into natural features such as forests and vegetation for they have a certain angle with their direction and azimuths, which reduced the accuracy of building extraction. Normalized circular polarization correlation coefficient is useful for distinguishing between buildings and natural features such as forests and vegetation. Therefore, this paper introduces the normalized circular polarization correlation coefficient, which is used as the polarization feature, and combines with the selected texture features to complete the image classification and extraction in order to improve the accuracy of building extraction. As shown in picture 2 :

\section{(2) Contrast}




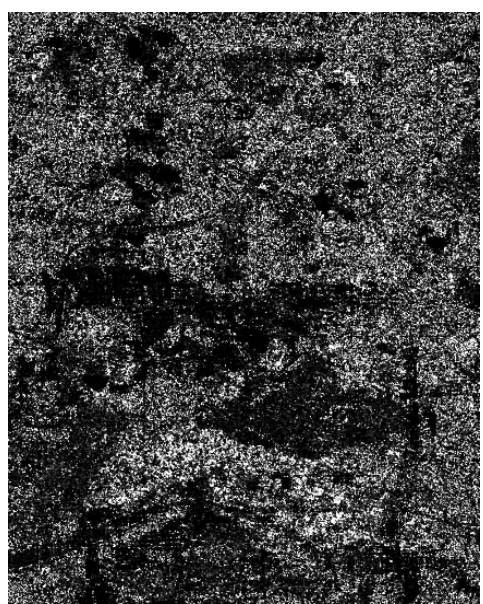

Figure 2. Normalized Circular-Pol Correlation Coefficient

\section{THE BUILDING EXTRACTION OF POLARIMETRIC SAR IMAGE}

\subsection{The selection method of texture feature}

The types and structures of buildings in urban areas are different, and the textures exhibited in polarized SAR images are also different. In Span map, the distinguishing between objects is relatively good, the structure and boundary contour of the features are clearer and more visible. The extracted texture features are easy to be detected and identified and the noise reduction effect of the Span image is obvious. Therefore, this paper uses Span image to extract texture features, as shown in Figure 3.

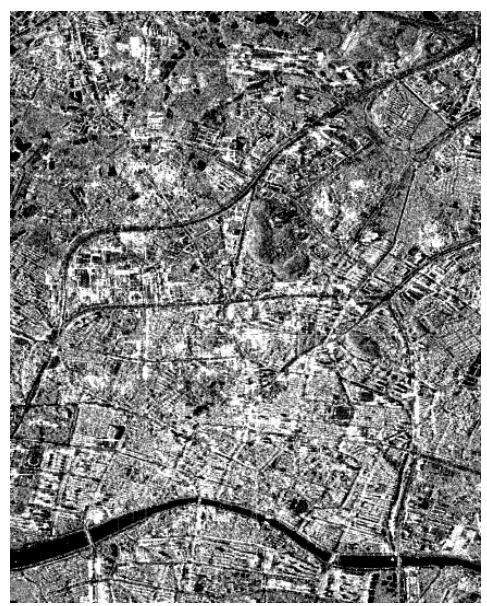

Figure 3. Span diagram

In order to reduce the amount of experimental calculation, this paper quantifies the Span image from 256 gray levels to 16 levels. Previous studies have shown that this processing step has little effect on the calculation of texture features based on gray level co-occurrence matrix (Deng, 2018). Then calculate the gray level co-occurrence matrix in four directions: $0^{\circ}, 45^{\circ}, 90^{\circ}$ and $135^{\circ}$, and finally use the average of the four directions as the eigenvalue. $\mathrm{d}$ is taken as 1 , the moving window is set to $7 \times 7$, the moving step size is a, b. And calculate the gray level co-occurrence matrix of the window area. Then move the texture window in turn, and repeat the previous processing steps, until each pixel of the image is calculated to obtain a complete texture image, thereby calculating the characteristic image of the polarized SAR image.

\subsection{The process of building extraction}

This paper proposes a method of building extraction of full polarization image of GF-3 based on associated feature and SVM. The technical flow of the method is shown in Figure 4. The specific steps are as follows: (1)Filtering SAR images to reduce the effects of speckle noise; (2) The gray-scale cooccurrence matrix method is used to extract the texture features of Span diagram, and the four kinds of texture feature statistics such as mean, entropy, contrast and homogeneity are calculated; (3) Principal component analysis is used to remove the correlation between them, and the first two texture features with the largest amount of information are selected; (4) The normalized circular-pol correlation coefficient is introduced as the polarization feature and combines with the best texture features; 5) Select training samples for both buildings and nonbuildings to train the SVM classifier;6)Input the feature image and complete the extraction of the building based on the trained SVM; (7) Accuracy evaluation of final building extraction results.

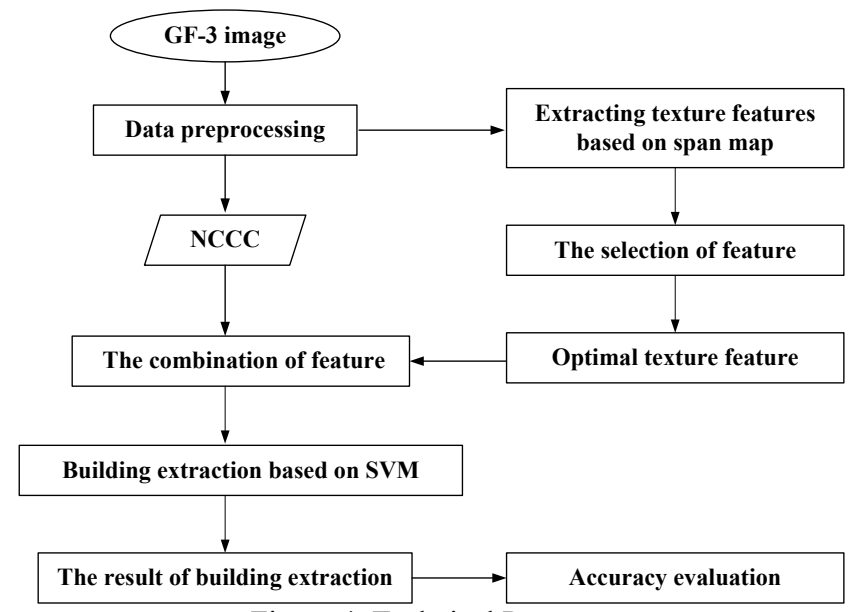

Figure 4. Technical Process

\section{DATA PROCESSING AND RESULT ANALYSIS}

\subsection{Experimental data and pretreatment}

This experiment uses full polarization image of GF-3 with a resolution of 8 meters. The study area is located in Guangzhou. This paper selects a sub-area of $1201 \times 1501$ as the experimental image, which mainly includes buildings, vegetation, water and so on. Firstly, the experimental image is preprocessed. In this paper, the image is filtered by Lee Refined filter of $7 \times 7$ window, as shown in Figure 5. It can be seen from the figure that the building has higher brightness on the GF-3 image, and has higher discrimination from other terrain texture features, and has strong directionality. 


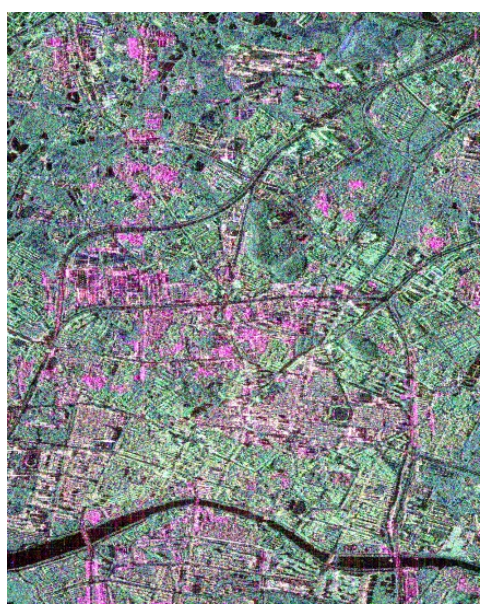

Figure 5. Filtered image

\subsection{The selection of texture feature}

In this paper, the texture features are calculated according to the method in Section 3.1, and four kinds of feature images of mean, entropy, contrast and homogeneity are obtained, as shown in the figure. Due to a certain degree of correlation between features, principal component analysis is used to remove the correlation between them, and the first two principal components with the largest amount of information (mean, entropy) are selected as the best statistic to describe the texture features.

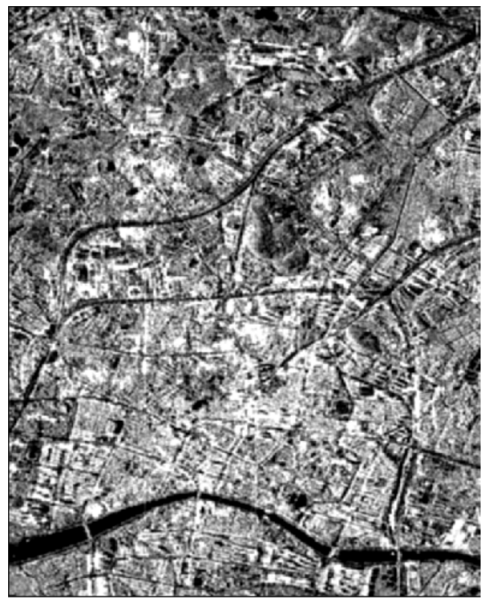

Figure 6. Mean

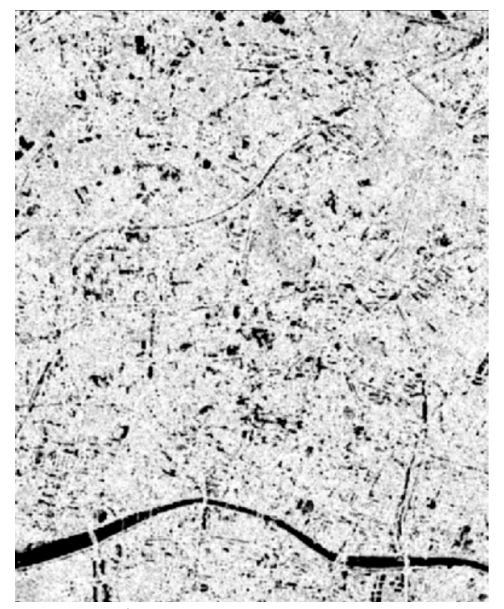

Figure 7. Entropy

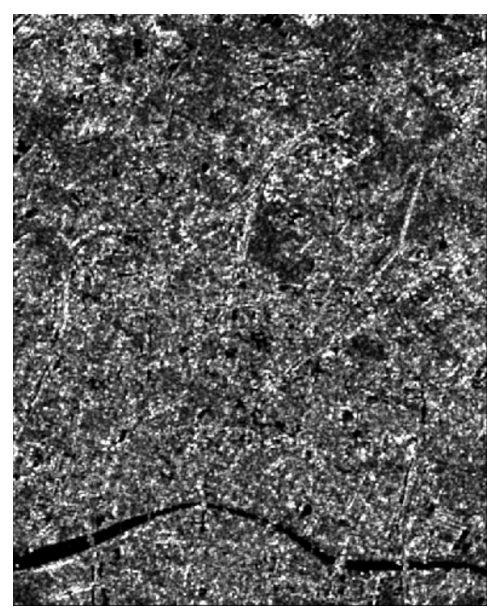

Figure 8. Contrast

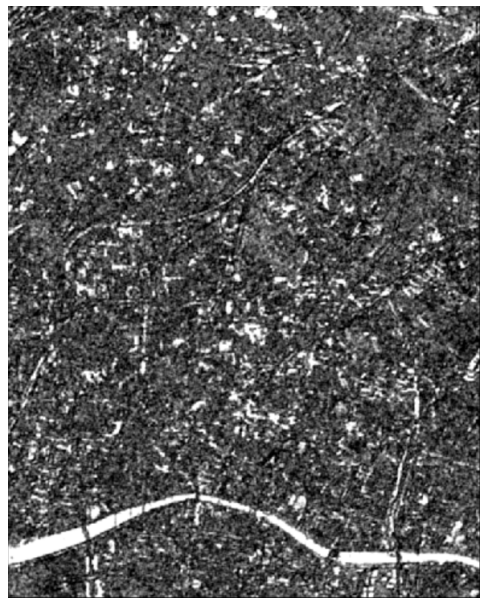

Figure 9. Homogeneity

\subsection{Equations, Symbols and Units}

The normalized circular-pol correlation coefficient is introduced and combines with the two principal components with the largest amount of information, and then the image is supervised and classified by SVM. The extraction result based on associated features and SVM as shown in the figure 10, and the extraction result using only texture features as shown in the figure 11 .

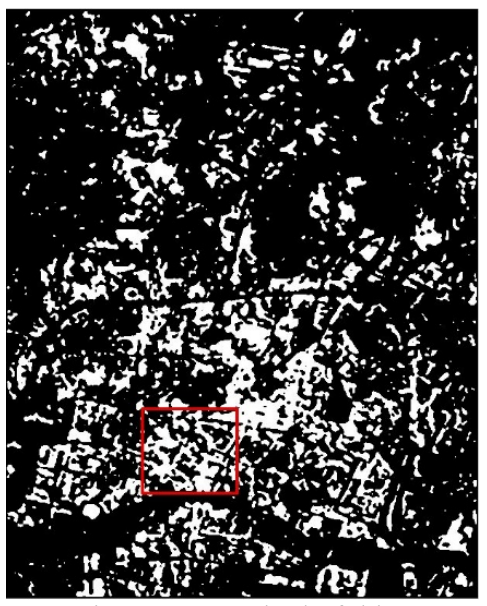

Figure 10. Method of this paper 


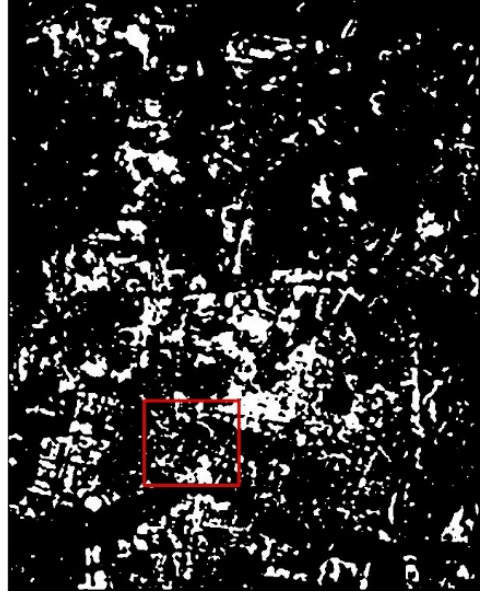

Figure 11. Texture method

As can be seen from Figure 11, the extraction of building is less effective compared with Figure 10. Many inclined buildings are easily misclassified into forest and vegetation, which reduces the extraction accuracy. In the picture, the red frame area is a phenomenon in which some buildings are mistakenly divided into vegetation. The introduction of the normalized circular polarization correlation coefficient obtains a better distinction between buildings and natural features such as forest and vegetation. From the above analysis, it can be seen that the associated polarization feature and texture feature can extract the building more accurately, so that the extraction result is optimized.

In order to better evaluate the classification results, this paper further evaluates the accuracy of the two extraction results, and calculates the confusion matrix, overall classification accuracy and Kappa coefficient of each category in the two extraction results. Accuracy evaluation as shown in Table 1:

\begin{tabular}{|c|c|c|c|c|c|}
\hline Method & Category & Building & $\begin{array}{c}\text { Non- } \\
\text { building }\end{array}$ & $\begin{array}{c}\text { Precision } \\
(\%)\end{array}$ & $\begin{array}{c}\text { Kappa } \\
\text { Coefficient }\end{array}$ \\
\hline \multirow{3}{*}{$\begin{array}{l}\text { Method } \\
\text { of this } \\
\text { paper }\end{array}$} & Building & 2048 & 22 & 98.94 & \\
\hline & $\begin{array}{c}\text { Non- } \\
\text { building }\end{array}$ & 592 & 7307 & 92.51 & \\
\hline & $\begin{array}{c}\text { Overall } \\
\text { accuracy } \\
(\%)\end{array}$ & & & 93.84 & 0.8301 \\
\hline \multirow{3}{*}{$\begin{array}{l}\text { Texture } \\
\text { method }\end{array}$} & Building & 5590 & 486 & 92.00 & \\
\hline & $\begin{array}{c}\text { Non- } \\
\text { building }\end{array}$ & 2523 & 15478 & 85.98 & \\
\hline & $\begin{array}{c}\text { Overall } \\
\text { accuracy } \\
(\%)\end{array}$ & & & 87.50 & 0.7019 \\
\hline
\end{tabular}

Table 1. Classification Confusion Matrix and Accuracy Evaluation

It can be seen from the table that there are many misclassifications when extracting buildings using only texture features, and the building extraction accuracy is relatively low. The overall classification accuracy is $87.5 \%$, and the Kappa coefficient is 0.7019 . Based on this method, the normalized circular polarization correlation coefficient is introduced as the polarization feature and combines with the best texture features to extract buildings. The experiment proves that the method of extracting buildings in this paper has higher precision, up to $93.84 \%$. There are fewer misclassifications, and the extraction of buildings is more accurate, and the extraction effect is better than the former method.

\section{CONCLUSION}

With the continuous development of SAR sensor technology, the resolution of high-resolution SAR images has also been greatly improved, and the application for urban building extraction is becoming more and more extensive. This paper extracts buildings in a certain area of Guangzhou based on GF-3 images. That provided technical support for GF-3 images in urban planning, urban land surveys, and geographic conditions monitoring applications. Due to the complex texture information of urban buildings in SAR images, the use of texture features alone cannot extract buildings better, and it is difficult to distinguish buildings and vegetation in the complex urban environment, this paper introduces the normalized circular polarization correlation coefficient as the polarization feature, combined with the texture features for image building extraction. The structure of the terrain and the boundary contour in the Span diagram are clearer, and the distinction between the objects in the image is better. Therefore, the texture features are extracted on the Span graph, and the correlation between them is removed by principal component analysis, then filter out the best texture features. Combine it with the normalized circular polarization correlation coefficient and extract buildings based on SVM. This paper also made a comparative experiment, the extraction results show that the proposed method can extract buildings more accurately, and the extraction accuracy is improved by nearly $6 \%$ compared with the use of texture feature extraction buildings. The extraction effect is obviously optimized.

\section{REFERENCES}

Ban Y., \& Hu H., 2007: RADARSAT Fine-Beam SAR Data for Land-Cover Mapping and Change Detection in the Rural-Urban Fringe of the Greater Toronto Area. 2007 Urban Remote Sensing Joint Event. doi:10.1109/urs.2007.371788.

Dell'Acqua $\quad$ F., $\quad \& \quad$ Gamba $\quad$ P., 2003. Texture-based characterization of urban environments on satellite SAR images. IEEE Transactions on Geoscience and Remote Sensing, 41(1), 153-159. doi:10.1109/tgrs.2002.807754.

Hu D.Y., Li X.G., Zhao W.G., \& Gong H.L., 2008. Texture Analysis and its Application for Single-Band SAR Thematic Information Extraction. IGARSS 2008 - 2008 IEEE International Geoscience and Remote Sensing Symposium. doi:10.1109/igarss.2008.4779149.

Deng H.R., Cui C.Y., Shan W.L., Xu M.Z., 2018. Urban Building Area Extraction Based on GF-3 Satellite SAR Images. Geomatics World, 25(06):79-84.

Haralick R. M., Shanmugam K., \& Dinstein I., 1973. Textural Features for Image Classification. IEEE Transactions on Systems, Man, and Cybernetics, SMC-3(6),610-621. doi:10.1109/tsmc.1973.4309314

Li Q., Zhang G.F., Gong L.X., Xue T.F., \& Jiang H.B.,2018. Extraction of earthquake-collapsed buildings based on correlation change detection of multi-texture features in SAR images. Journal of Remote Sensing,22(S1):128-138.

Sun P.,2013. Research on Building Extraction Method of Polarimetric SAR Image. Capital Normal University,2013. 
Tison, C., Nicolas, J.-M., Tupin, F., \& Maitre, H., 2004. A new statistical model for Markovian classification of urban areas in high-resolution SAR images. IEEE Transactions on Geoscience and Remote Sensing, 42(10), 2046-2057. doi:10.1109/tgrs.2004.834630.

Xu J., Chen Y.Y., Huang Q.H., He X.F., 2012. Built-up Areas Extraction in High Resolution Spaceborne SAR Image based on the Integration of Gray and Texture Features. Remore Sensing Technology and Application, 2012,27(05):692-698.

Yang J., Zhao L.L., Li P.X., Lang F.K., 2012. Preserving Polarimetric Scattering Characteristics Classification by Introducing Normalized Circular-pol Correlation Coefficient. Geomatics and Information Science of Wuhan University, 2012,37(08):911-914+935+880.

Zhao L.J., Gao G., Kuang G.Y., 2009. Variogram-based Builtup Areas Extraction from High-resolution SAR Images. Journal of Signal Processing, 2009,25(09):1433-1442.

Zhai W., Shen H.F., Huang C.L, 2016. Collapsed Buildings Extraction from the PolSAR Image based on the Analysis of Texture Features. Remore Sensing Technology and Application, 2016,31(05):975-982. 